\title{
Creencias del profesorado de Educación Básica en formación sobre la enseñanza de la ciencia escolar: Análisis desde un debate de grupo*
}

\author{
Beliefs of elementary education future teachers of the Science teaching: A group dis- \\ cussion analysis
}

\section{Crenças do professorado da Educação Básica em formação sobre o ensino da Ciência escolar: análises a partir de um debate em grupo}

\author{
Eduardo Ravanal Moreno, ${ }^{\text {a }}$ Mario Quintanilla Gatica ${ }^{\mathrm{b}}$ \\ aUniversidad Central. Telf.: (02) 5826640. Correo electrónico: 1ravanalm@ucentral.cl \\ bPontificia Universidad católica de Chile. Telf.: (02) 6865361. Correo electrónico: mquintag@puc.cl
}

\begin{abstract}
RESUMEN
La formación de profesores en Chile es un tema de preocupación pública, por ello, la investigación de y sobre los procesos de formación y desarrollo profesional docente son interesante e importantes de abordar. Con el propósito de identificar y caracterizar las concepciones del profesorado de Educación Básica en formación, se diseña un grupo de debate en el marco de un programa de formación docente en el que participan tres profesoras en formación. El análisis de las interacciones dialógicas se realiza usando segmentos de interactividad. Algunos de nuestros resultados revelan que: Existe fragmentación teórica sobre el conocimiento didáctico de la disciplina que se enseña; y se aprecia una sobrevaloración del libro de texto como dispositivo de información e insumo de actividades de aprendizaje. Finalmente, sostenemos que es necesario intensificar el diseño de sesiones de reflexión con el profesorado en formación para la promoción de conocimiento profesional.
\end{abstract}

Palabras clave: profesor de educación básica, concepciones, actividades de aprendizaje, libro de texto.

\begin{abstract}
Teacher training in Chile is (currently) a matter of public concern, thus, research on and of teacher training processes and professional development are (becoming more) interesting and important to address. In order to identify and characterize Elementary School teacher trainee's conceptions, a debate group is set up in the framework of a teacher training program in which three teacher trainees attend. The analysis of dialog exchanges is carried out using segments of interactivity. Some of our results reveal that: There is theoretical fragmentation over the knowledge of didactics in the discipline being taught. It is also observed, that textbooks, as information (carrier) devices and input for learning activities, are overrated. Finally, we argue that it is necessary to intensify the design of reflection meetings on teacher trainees in order to promote professional knowledge.
\end{abstract}

Key words: elementary school teacher, conceptions, learning activities, textbook.

\section{RESUMO}

A formação de professores no Chile é um tema de pública preocupação; por isso, a pesquisa de/sobre processos de formação e desenvolvimento profissional docentes são importantes e interessantes de ser abordada. Para identificar e caracterizar as concepções dos professores de Educação Básica em formação, organizou-se um grupo de debate

* El trabajo se hace parte del proyecto CONICYT/AKA 04 '"Desarrollo de habilidades y competencias de pensamiento científico en estudiantado y profesores" (abrev.) que dirige el Dr. Mario Quintanilla Gatica, académico del Departamento de Didáctica de la Facultad de Educación de la Pontificia Universidad Católica de Chile (PUC). Una versión preliminar de este trabajo fue presentado en la 4ta Jornada Internacional de Investigadores en Educación y X Jornada Nacional de Investigadores en Educación, Osorno, Chile. 
no qual participam três professoras em formação em um programa de formação de professores. As análises das interações dialógicas se realizam usando segmentos de interatividade. Alguns dos resultados revelam que: existe fragmentação teórica sobre o conhecimento didático referente à disciplina que se ensina; também se identifica uma supervalorização do livro como um instrumento de informação e como recurso para atividades de aprendizagem. Finalmente, defende-se que é necessário intensificar a estrutura das sessões de debates com os docentes em formação para promover o conhecimento profissional.

Palavras chave: professor de educação básica, conceitos, atividades de aprendizagen, livros didáticos.

\section{INTRODUCCIÓN}

La formación de profesores en Chile es, hoy, un tema de preocupación pública; la búsqueda incesante de modelos teóricos y metodológicos que permitan entender los procesos implícitos en la enseñanza para la promoción de aprendizajes de calidad que convoquen a niños, niñas y jóvenes a participar activamente en la sociedad, nos lleva a discutir y a debatir sobre el modo en que debemos enfrentar esta tarea. Sin embargo, como plantea Gorodokin (2006), abordar la formación de formadores es una reflexión compleja de pensamiento y acción que nos invita a una práctica paradigmática para conocer y comprender el conocimiento profesional construido y en construcción. Desde estas inquietudes, el objetivo de la investigación es identificar y caracterizar las concepciones sobre la enseñanza de la ciencia escolar para niños y niñas de 9 a 10 años declaradas por tres profesoras en formación de Educación Básica y analizar en qué medida estas concepciones dan cuenta de su conocimiento didáctico.

\section{FORMACIÓN DOCENTE Y SU CONTEXTO}

La formación docente implica, irreduciblemente, un proceso de práctica profesional inicial que en muchas universidades se han programado a los largo de toda la carrera docente. Ahora bien, particularmente el último año de carrera, los profesores en formación abordan una asignatura denominada, tradicionalmente, práctica profesional (PP). Un estudiante de pedagogía que cursa su PP es atendido por un profesor universitario y otro propio del establecimiento educacional donde se desarrolla la praxis.

Al respecto, los primeros alcances. Comúnmente, el profesor universitario centra su rol en el aprendiz y es el responsable principal de cómo el estudiante en práctica explora y aborda las instancias propias de la asignatura. Como sostiene Vélaz de Medrano (2009: 213), "un profesor tutor de práctica profesional evalúa la situación del tutelado, le plantea problemas, evalúa su desempeño y vuelve a plantear tareas para reforzar y extender su aprendizaje". Particularmente, pensamos que las orientaciones derivadas de la relación profesor tutor y estudiante en práctica deben transitar hacia un espacio diverso que promueva el conocimiento didáctico del contenido disciplinar (CDCD) para fortalecer el conocimiento profesional (Gutiérrez, Valbuena y Neira 2009), dado que el primero permite la organización de los saberes escolares con un estatus epistemológico diferenciado (Valbuena, 2007). Lo anterior lleva a plantear que no es pertinente referirse a un profesor tutor, sino, más bien, situar la discusión desde un profesor mentor que orienta su trabajo hacia el desarrollo de competencias profesionales docentes como lo plantea Vélaz de Medrano (2009). La propuesta nos parece muy interesante, considerando que la construcción de conocimiento profesional implica, entre otras cosas, hacerlo explícito y 
relevarlo a niveles de compresión conceptual, procedimental y contextual desde la reflexión en la acción con el propósito de favorecer la transformación de las prácticas docentes; en este marco conceptual, compartimos la idea de Bertelle et al. (2006), quienes sostienen que el conocimiento profesional se construye por la reelaboración de los distintos saberes en contextos distintos y epistemológicamente diferenciados, lo cual implica resignificar los protocolos declarados y desarrollados en la actualidad por distintos equipos de práctica, caracterizados por nociones tutoriales de tipo instrumental, en que se desarrolla una asistencia técnica centrada en identificar errores o imprecisiones más que en la promoción de espacios de reflexión para la resignificación propia de la formación docente.

Ahora bien, la enseñanza, aprendizaje y evaluación de la ciencia es de interés y preocupación para el sistema educacional chileno; los resultados de pruebas estandarizadas nacionales e internacionales revelan la complejidad de aprender ciencias, por ello, los aportes en investigación educativa al respecto son un valioso aporte a la comunidad científica y al profesorado en formación o en ejercicio para la toma de decisiones hacia una buena enseñanza de la ciencia escolar. Ésta, en su constructo, propone la conexión entre la teoría y los hechos del mundo; bajo este foco, compartimos que la ciencia escolar busca entender la relación entre los contenidos científicos y la manera de enseñarlos y aprenderlos, y dar respuesta a los problemas que se generan a partir de su puesta en marcha en una relación dinámica entre ellos (Orellana, 2008). Ahora bien, la escuela debe ser, irreduciblemente, el sitio privilegiado que enseña a interpretar los hechos o fenómenos -naturales o artificiales- a partir de la promoción de un "hacer y pensar" teórico (Ravanal y Quintanilla, en prensa); esto implica desafíos y cambios epistemológicos y didácticos en la actuación pedagógica del profesorado, particularmente, si consideramos que:

a) Los conocimientos que adquieren los profesores en la formación inicial tienen fecha de caducidad (Marcelo, 2002).

b) El conocimiento sobre la enseñanza es de carácter tácito, difícil de sistematizar (Moreno, 2005).

c) El conocimiento distribuido, por complejo, ningún profesor lo posee completo (Putnam y Borko, 2000).

Lo anterior, sin duda, es una alerta y, a la vez, un compromiso que nos lleva a abordar el cómo enfrentar la formación profesional docente para preparar futuros profesores; conscientes de su conocimiento profesional, capaces de autorregular su saber profesional en pos de una enseñanza con sentido y valor para los estudiantes; sobre todo si entendemos que son ellos quienes deciden qué enseñar; cómo y cuándo hacerlo (García y Angulo, 2003). Enfrentar este reto implica comenzar a explorar las representaciones de los profesores tutores/mentores sobre la promoción y desarrollo de conocimiento profesional docente (CPD); como de los protocolos que se definen para dichos propósitos en relación estrecha con el aprender a enseñar desde la reflexión profunda y no reduccionista; que sólo atiende a identificar problemas y limitaciones en la actuación docente (Kelchtrermans, 2001).

Es importante reconocer que el profesorado en formación aborda este proceso de orientación profesional bajo la dirección de dos tipos de profesores-tutores, el representante de la Universidad y el responsable de acompañarlo en el establecimiento educacional. Es interesante lo anterior, dado que se identifican dos profesores para orientar a un único profesor en formación; sin embargo, no existen vínculos de trabajo colaborativo entre 
ellos que favorezcan y potencien la promoción de competencias profesionales, es más, cada profesor, aborda la enseñanza-aprendizaje para el profesor en formación y discute sobre ella, desde un enfoque más bien pragmático e individual.

Este hecho de realidad es una condición irreductible que nos lleva a promover conocimiento profesional desde una reflexión profunda, que dé pistas a los profesores en formación sobre los ámbitos implícitos y explícitos que se ponen en juego en la enseñanza, aprendizaje y evaluación de una disciplina, de manera que pueda regularlos.

Esta situación, que aparentemente suele ser una generalidad en las escuelas de Educación, nos lleva a sugerir que los procesos de práctica pedagógica (PP) atiendan a los siguientes atributos:

a) Estar inserta bajo un modelo didáctico para la formación inicial del profesor (García y Angulo, 2003).

b) Entenderlas como un proceso altamente reflexivo, práctica reflexiva (Gutiérrez, Valbuena y Neira 2009).

c) Constituir equipos de profesores mentores (Vélaz de Medrano, 2009) que orienten el proceso en un marco de desarrollo profesional.

Consolidar equipos auténtico de trabajo para la orientación y regulación de los procesos implicados en la PP que garanticen la formación de 'buenos profesores', es un desafío y, por cierto, una preocupación real para el Estado de Chile; por ello, diseñar e implementar una metodología de trabajo de PP que involucre a todos los profesionales responsables (tutor universitario y del establecimiento educacional) del proceso de formación docente bajo los lineamientos de un modelo didáctico, nos parece un aporte interesantísimo, sobre todo cuando se piensa en consolidar equipos de trabajo que fortalezcan el conocimiento profesional desde la reflexión sobre la acción, en ambientes intencionados y contextualizados. La inclusión de los profesores en los centros educativos, que asisten a los estudiantes en práctica, y que desarrollan su carrera docente en las distintas escuelas y liceos, es una apuesta interesante para mejorar procesos de formación profesional, dado que verán en ello un real espacio de participación, crecimiento y resignificación de su hacer docente. En ese plano, Tardif (2004: 169) sostiene que "un profesor es un sujeto que asume su práctica a partir de los significados que él mismo le da, un sujeto que posee conocimiento y un saber hacer provenientes de sus propia actividad y a partir de los cuales la estructura y la orienta".

Lo anterior exige en el profesorado un tipo de conocimiento que lo distingue de otros profesionales: éste es su conocimiento didáctico (CD). El CD permite la construcción de conocimiento y significados. Sin embargo, dicho conocimiento, en muchas ocasiones, se invisibiliza en el profesor en formación, sustentando su actuación en la experiencia o el sentido común. Creemos que estas creencias deben ser una línea de discusión en y durante su práctica profesional, desde un programa de mediación y reflexión en la acción, asunto que nuestro equipo ha estado desarrollando desde el año 2007 con profesores en ejercicio.

\subsection{LA REFLEXIÓN Y EL DESARROLLO PROFESIONAL DOCENTE}

La formación de profesores implica hoy entenderlo como un proceso de desarrollo que favorece la construcción de su saber profesional. En ese contexto, nos parece interesante 
la propuesta de García (2009: 78), que dice: "La formación del profesorado no hay que plantearla como un "cambio", sino más bien como un proceso interno de "crecimiento" y de "desarrollo" gradual a partir de lo que ya piensan y hacen..." Desde ahí, la formación profesional como un proceso de desarrollo conlleva a la toma de conciencia sobre los tránsitos, transformaciones y eventuales cambios en el sistema de ideas del profesor (Perafán, 2005; Ravanal y Quintanilla, en prensa), los cuales condicionan, en gran parte, la actuación pedagógica. Por ello, un proceso de formación docente desde un plano de desarrollo, implica asumir que la reflexión de y sobre la práctica pedagógica son espacios diversos metacognitivamente que contribuyen al ámbito personal de conocimiento y estructuras interpretativas de la profesión docente (García, 2009). Desde esa perspectiva, orientar la formación del profesorado como proceso de desarrollo nos parece interesante un punto importante de discusión en el marco de la reflexión como recurso para aprender a enseñar (Fuentealba y Galaz, 2008). Por lo tanto, es necesario fortalecer líneas de investigación referidas a la reflexión en y para la formación docente, de manera que podamos contribuir resignificando el imaginario del profesor en formación. Por ello, el propósito de esta comunicación es explorar las concepciones del profesorado de educación básica en formación sobre la enseñanza de la ciencia escolar; información que nos permite aproximarnos a nuevos modos de abordar los procesos de acompañamiento docente, definidos específicamente en la práctica profesional.

\section{DISEÑO DE INVESTIGACIÓN}

La investigación se orienta desde un paradigma de investigación interpretativo y transformador, como ya ha sido trabajado en otras investigaciones por nuestro grupo de investigación (Ravanal, 2009; Cuellar, 2010; Camacho, 2010). Su propósito es identificar y caracterizar la narrativa del profesorado en formación, dando cuenta del sistema de creencias sobre la enseñanza de la ciencia desde un marco multidimensional y aproximándonos a los niveles de comprensión del 'pensamiento del profesor de Educación Básica' sobre la enseñanza.

\subsection{PARTICIPANTES}

En el marco de un programa de formación convocamos a tres profesoras de Educación Básica a participar de un debate de grupo sobre el diseño de la enseñanza de las ciencias para niños y niñas de 9 a 10 años de edad. Las profesoras en formación invitadas fueron elegidas por su interés personal en discutir temas vinculados con la enseñanza de la ciencia que se manifiesta por desarrollar, durante el año 2010, su seminario de investigación en esta línea teórica, además de estar en su último año de carrera. En términos curriculares, las participantes han cursado su práctica profesional y el taller de reflexión de y sobre ésta. En ese contexto, destacamos que el proceso de práctica profesional es una instancia académica desarrollada en un centro educativo y las sesiones de reflexión son realizadas en la universidad, ambos espacios de desarrollo coordinados por un profesor de la Escuela de Educación Básica. 


\subsection{METODOLOGÍA DE RECOGIDA Y ANÁLISIS DE INFORMACIÓN}

La recogida de información se realizó con un enfoque etnográfico desde un debate de grupo coordinado y moderado por el autor del artículo. Las interacciones dialógicas derivadas de la reflexión fueron videograbadas y transcritas total y literalmente. La información que presentamos corresponde a una sesión de trabajo vinculada con la enseñanza de la ciencia para niños y niñas de educación básica. Se programa sólo una instancia de debate, dado que se pretende poner en juego el conocimiento profesional docente en desarrollo desde el sistema de ideas integradas que determinan la actuación pedagógica y didáctica de un profesor.

El análisis de las interacciones dialógicas se realiza usando Segmentos de Interactividad (Márquez, 2002; Couso y Pinto, 2009) para identificar unidades de reflexión docente relevantes para la investigación. Los segmentos de interactividad se dividen según núcleos temáticos paradigmáticos derivados del grupo de debate y de interés para la investigación, como lo muestra la Tabla 1.

\begin{tabular}{|c|c|}
\hline Segmento de Interactividad & Turno de habla \\
\hline Reflexión en torno a la enseñanza de una noción científica & $01-141$ \\
\hline Atributos y actividades según momentos de una clase de ciencias & $142-404$ \\
\hline Formulación de preguntas en la clase de ciencias & $273-314$ \\
\hline
\end{tabular}

Tabla 1. Segmentos de interactividad derivados del debate de grupo con profesoras en formación de Educación Básica

Posteriormente, se realizó un análisis cualitativo del contenido de las narrativas docentes derivadas de las interacciones dialógicas de y entre los participantes durante el grupo de debate. Finalmente, realizamos una categorización (Rodríguez et al., 1999) de los aspectos conceptuales implícitos en la narrativa del profesor en formación a partir de la segmentación de una narrativa para un fragmento de interactividad.

\section{RESULTADOS}

Los resultados de la investigación intentan abordar la noción de aprendizaje, enseñanza, atributos y propósitos de las actividades, según momentos de clase y formulación de preguntas. Los resultados relacionados con evaluación de la ciencia escolar serán presentados en un trabajo posterior que estamos preparando. Desde un análisis de contenido semántico y con el propósito de interpretar la realidad sobre el diseño de la enseñanza de la noción de materiales en la clase de ciencias naturales, comentamos que:

\subsection{REFLEXIÓN EN TORNO A LA ENSEÑANZA DE UNA NOCIÓN CIENTÍFICA}

El análisis para este segmento de interactividad comprende 3 aspectos, que son: orientación a la tarea, criterios de selección para un dispositivo de enseñanza y las concepciones del profesor en formación sobre el rol del libro de texto en la enseñanza. 


\section{a) Orientación a la tarea escolar}

El aprendizaje exige el diálogo entre el punto de vista del profesor y el punto de vista del estudiante (Márquez et al., 2004) para favorecer la construcción de significados. En ese contexto, es relevante considerar que la actuación del profesor no debe invisibilizar en su discurso la orientación a la tarea. Sin embargo, estos asuntos no son concientes en el profesor en formación; lo que restringe la regulación y gestión en aula para el aprendizaje. En muchas ocasiones, el profesor en formación asume que las instrucciones, dispositivos de enseñanza, como los propósitos de las tareas para los escolares, son suficientemente claros en sí mismos (Figura 1). Es preocupante el mermado análisis didáctico de y sobre la formulación de preguntas. En espacios problemáticos sobre la enseñanza, la formulación de preguntas es urgente si no hay una definición fundamentada del tipo y el rol de ellas para promover la enseñanza de una noción científica en particular.

59. C: la profesora proyecta una imagen a través de un power point...

60. Pf1: si

61. C: la imagen

62. Pf1: no, tenemos que dar las instrucciones de que las observen

63. C: ¿cuáles son las instrucciones? ¿Hay preguntas de por medio?

64. Pf1: si

65. Pf2: si, porque vamos ir mostrando varias imágenes, por ejemplo observen la imagen, mencionen los objetos que ven, cómo están hechos, de qué están hechos, cuál es su uso.

Figura 1. Transcripción de una actividad de inicio para la enseñanza de los materiales a estudiantes de cuarto año básico. (Pf1: profesor en formación 1; C: coordinador)

\section{b) Criterios de selección para un dispositivo de enseñanza}

El profesorado en formación decidió usar una imagen en el momento de inicio de la clase de ciencias para abordar la enseñanza de los materiales. En ese contexto, problematizamos el tipo de imagen que se desea compartir en el aula para promover el aprendizaje. Desde ahí, la información derivada de los registros de habla del profesorado en formación nos lleva a afirmar que el conocimiento didáctico sobre dispositivos de enseñanza es un tipo de conocimiento fragmentado que transita por ámbitos más bien genéricos e instrumentales. Existe una imagen ingenua sobre el "uso didáctico" de los dispositivos de enseñanza; definitivamente, se hace visible el rol de ellos en la estructura de recurso didáctico, pero no los protocolos de uso para promover el aprendizaje; en ese marco, no emerge la didáctica del recurso. Desde esa perspectiva, aparece una imagen ingenua de la didáctica, como plantea Quintanilla (2003). Ahora bien, desde el punto de vista epistemológico, la enseñanza se orienta desde una visión tradicional, centrada en el producto y en un aprendizaje por apropiación. Estos antecedentes coinciden con 
otros estudios que hemos realizados con profesores de biología en ejercicio (Ravanal y Quintanilla, 2010).

67. C: la imagen no puede ser al azar

68. Pf:no

69. C: tiene que seleccionar una buena imagen. A su juicio ¿cuál sería el criterio para elegir esa imagen en términos pedagógicos? Porque pueden haber miles, pero ¿cuál? No sé, pero ¿por qué elijen esa y no otra?

70. Pf1: a tienen que poner más claro que contenga todos los elementos que nosotras vamos a ver, los materiales que queremos ver.

71: C: ya

72: Pf2: yo creo también...

73 Pf3: //interrumpe// que lo conozcan los alumnos yo creo, que sea de su experiencia.

Figura 2. Transcripción de una actividad de inicio para la enseñanza de los materiales a estudiantes de cuarto año básico vinculado con la toma de decisiones sobre dispositivos de enseñanza (Pfx: profesor en formación 1, 2 ó 3; C: coordinador).

\section{c) Concepciones referidas al libro de texto}

El profesor de EGB en formación asigna un rol importante y relevante al libro de texto, particularmente, en lo referido a qué y cómo enseñar. En ese sentido, la toma de decisiones del profesorado en formación está determinada por la propuesta del libro de texto más que por el saber profesional que debiera orientar la toma de decisiones al respecto.

“...tenemos, ¿cómo se llama? El libro del profesor, de ahí está la guía y materiales, ¿lo quiere ver? (Pf1: 13).

“... presentar power point... con imágenes... parecidas a las del libro” (Pf2:32).

Es preocupante que profesores en procesos de finalización de su carrera docente, orienten la toma de decisiones desde ámbitos instrumentales-operativos carentes de sustento teórico sobre qué y cómo usar un recurso para promover la enseñanza que diseñan. La sobrevaloración del libro de texto como de las orientaciones ministeriales merman espacios reales de discusión y debate del rol del profesor; en ese sentido, los formadores de profesores y específicamente los responsables de coordinar y liderar procesos de desarrollo profesional, como los profesores de práctica pedagógica por ejemplo, que disponen de espacios de reflexión reales, reorienten el discurso hacia la construcción de saberes pedagógicos y didácticos de modo de transitar hacia espacios auténticos de formación docente:

“... y entre los criterios también, ¿ sirve... por ejemplo que el hecho de que los niños tengan el texto, porque no podemos pedir más sino van a llegar?” (Pf2:19).

"[Por qué piensan que es una buena actividad]... porque las propone el MINEDUC (Pf1:233)... están en el texto de estudio y todos los alumnos lo tienen" (Pf1: 235). 
La enseñanza de una disciplina debe promover la construcción de significados y conocimiento; esto obliga al profesor saber regular y autorregular el proceso de enseñanza, aprendizaje y evaluación; propósitos que implican conocimiento didáctico del contenido disciplinar, en ese sentido, un saber profesional que permita seleccionar un dispositivo de enseñanza considerando múltiples dimensiones y no sólo orientar la decisión o selección desde una perspectiva operativa, como lo deja entre ver las siguientes textualidades:

“... sí poníamos, por ejemplo, primero la imagen de un paisaje, por ejemplo, como salía en el libro" (Pf1:38).

“... en el libro van a trabajar los materiales” (Pf1:109).

“... hay preguntas, lo que pasa es que en el mismo libro está el disfraz, está cortita” (Pf1:2007).

“[las preguntas que van a proponer]... también están en el libro2 (Pf1:211).

"Desarrollan las actividades de la página 60, 61 y 62 del libro" (Pf1:223)

El uso del libro de texto, a juicio del profesor de EGB en formación, es insumo de actividades de aprendizaje, fuentes de preguntas para los estudiantes, crédito indiscutible del contenido en discusión. Todas estas creencias son contrarias a la formación de un profesor capaz de proponer criterios de selección, implementación y evaluación de cualquier dispositivo de enseñanza. Estos antecedentes son un real desafío para el formador de profesores, quienes debemos intensificar la formación de y hacia el conocimiento profesional y, específicamente, el conocimiento didáctico de la disciplina, de lo contrario, nunca abandonaremos la postura ingenua de la enseñanza y la importancia que la experiencia tienen en los cambios del profesor, cambios que ocurren si, y sólo si, se toma conciencia de qué y cómo se hacen las cosas, en este caso, una clase de ciencias.

\subsection{ATRIBUTOS Y ACTIVIDADES SEGÚN MOMENTOS DE UNA CLASE DE CIENCIAS DESDE LA PROPUESTA DE ACTIVIDADES DE APRENDIZAJE}

Lo primero que deseamos compartir es que el profesorado en formación, orienta su diseño de clase en tres momentos: inicio, desarrollo y cierre. En la tabla 2 se resumen las representaciones de los docentes en formación sobre los atributos y rol de los momentos de una clase discutidos desde las actividades de enseñanza que se pueden proponer para la enseñanza de los materiales.

\begin{tabular}{|c|c|c|}
\hline $\begin{array}{c}\text { Momentos de una clase } \\
\text { de ciencias }\end{array}$ & $\begin{array}{c}\text { Atributos de las } \\
\text { actividades según } \\
\text { momento }\end{array}$ & $\begin{array}{c}\text { Propósitos de las } \\
\text { actividades según momento }\end{array}$ \\
\hline INICIO & $\begin{array}{c}\text { - Actividad breve. } \\
\text { - De carácter lúdica. }\end{array}$ & $\begin{array}{c}\text { - Motivación } \\
\text { Generar interés y el } \\
\text { aprendizaje } \\
\text { P Presentar el tema } \\
\text { - Involucrar a los estudiantes }\end{array}$ \\
\hline
\end{tabular}




\begin{tabular}{|c|c|c|}
\hline$\bullet$ DESARROLLO & $\begin{array}{c}\bullet \text { Las actividades son } \\
\text { extensas y } \\
\bullet \text { Lúdicas }\end{array}$ & $\begin{array}{c}\text { - Los estudiantes aplican, } \\
\text { tratan con el contenido... } \\
\text { Trabajan directamente. } \\
\bullet \text { Poner en práctica el } \\
\text { contenido } \\
\text { El profesor presenta el } \\
\text { contenido }\end{array}$ \\
\hline CIERRE & No aparecen propuestas & $\begin{array}{c}\text { E Evaluación: guía, } \\
\text { observación directa, } \\
\text { preguntas }\end{array}$ \\
\hline
\end{tabular}

Tabla 2. Representaciones de los profesores en formación sobre los atributos y propósitos de una actividad de inicio, desarrollo y cierre

Al respecto, percibimos que cada una de las características o propósitos que definen un diseño para cada momento de clase, es declarado en términos genéricos e ingenuos. Según la evidencia, el profesor en formación ha asumido ciertas conceptualizaciones, que impiden una reflexión profunda de y sobre la enseñanza que desean promover. Particularmente, extrañamos argumentos para la toma de decisiones, desde esa perspectiva, el saber profesional no es transferido a situaciones complejas, como el diseño de clase. Destacamos que las actividades propuestas por el profesor en formación, en muchas ocasiones, son situadas en un momento u otro, más bien, por su finalidad que por sus atributos; lo que resta, irreduciblemente, al diseño y selección de actividades; es más, orientar estos procesos desde los propósitos implica asumir un obstáculo en la enseñanza, dado que la asignación de una actividad de aprendizaje está mediada por la representación que de ésta tenga el profesor sobre la finalidad que persigue.

\section{ACTIVIDADES DE INICIO}

147. Pf2: se supone que hay que presentar // como una presentación al tema, que motive al alumno, que despierte los aprendizajes previos de ellos. 148. C: ya

149. Pf2: que los involucre en lo que voy a enseñar toda la clase 150. C: O sea las actividades de inicio tienen como propósito generar la motivación, generar interés.

151. Pf2: si

153. Pf2: siempre nos ha dicho que debe ser una presentación no muy extensa, o sea, de cinco o diez minutos.

154. C: Ya

155. Pf2: en general lúdicas 
156: Pf3: que participen (los alumnos)

ACTIVIDADES DE DESARROLLO

189. Pf2: son extensas

190. C: ya

191. Pf2: es como en práctica lo que se trató de enseñar en la motivación.

192. Pf1: no

193. Pf3: lo que se quiere lograr en la clase. Puede ser como algo que se quiere lograr en la clase final.

194. C: Ya

195. Pf3: trabajarlo // lograrlo

196. Pf2: se trabaja el contenido y se profundiza

197. C: ahí

198. Pf1: se supone que se presenta el contenido. Se trabaja el contenido.

\section{ACTIVIDADES DE CIERRE}

Luego de revisar la planificación propuesta el coordinador plantea:

360. c: La última etapa dice cierre y evaluación (lee la planificación) ¿en qué consiste la última etapa.. aquí no indican actividades...

361. Pf1: es que es la matriz de la Universidad

.

367. Pf1: pueden haber actividades, de hecho que haya una guía puede evaluar con una guía o haciendo preguntas.

368. Pf2. Es una observación directa más que nada.

369. C: y una observación directa ¿qué sería, una actividad o una evaluación? 370. Pf2: sería como van desarrollando la clase o las actividades a lo largo de la clase si lo están haciendo bien.

Figura 3. Transcripción de un diálogo referido a los atributos y propósito de una actividad de inicio, desarrollo y cierre (Pfx: profesor en formación 1,2 ó 3; C: Coordinador).

\subsection{FORMULACIÓN DE PREGUNTAS}

Las preguntas son dispositivos de enseñanza interesante e importante para promover el aprendizaje de la ciencia en la escuela. Sin embargo, poco cuidado hemos puesto al respecto; es más, creemos que existe escasa reflexión sobre ello. Algunos estudios (Roca, 2005; Márquez et al., 2004) han intentado ofrecer un corpus de conocimiento sobre las preguntas y el aprendizaje de la ciencia en la escuela. Uno de nuestros estudios ha 
demostrado que el profesorado en formación formula preguntas en un plano instrumentaloperativo con el propósito de recoger información de carácter memorístico. Son muy pocas las preguntas que promueven la elaboración personal de posibles respuestas a las preguntas que plantea un profesor en formación. Ahora bien, en este estudio particular, hemos evidenciado que las preguntas son un saber y saber hacer implícito en la actuación pedagógica; asunto que no obliga planear las preguntas previo abordaje de la enseñanza. Algunas textualidades derivadas del debate de grupo son:

“...en general nunca escribimos las preguntas” (Pf2:166)

"nunca habíamos pensando en: ¿qué nos van a responder? ¿Qué me va a responder? ¿Me va a decir lo que yo quería saber?” (Pf2:170)

Esa cuestión nos lleva a pensar en una visión tradicional de enseñanza, centrada en el producto y no en espacios auténticos de co-construcción desde la propuesta de "buenas" preguntas. Junto con ello, el profesor en formación no está conciente de la importancia que tienen las representaciones iniciales en el aprendizaje de la ciencia; y cómo las preguntas contribuyen a ello. Algunas de estas ideas serán difundidas en una nueva comunicación que estamos preparando.

\section{REFLEXIONES FINALES}

La enseñanza de la ciencia se aborda poniendo en común creencias; desde ahí que las orientaciones a la tarea que promueva el profesorado son importantes y relevantes para promover el aprendizaje. Una orientación a la tarea implica generar espacios de autorregulación que movilicen a los sujetos en distintos planos de desarrollo (Labarrere y Quintanilla, 2002), de manera que pueda analizar las situaciones para comprenderlas.

La creencia de que las instrucciones, las llamadas de atención, los contenidos disciplinares en juego son 'entendidos' por todas y todos los estudiantes, restringe posibilidades docentes de conocer cómo aborda una tarea los estudiantes; así como restringe posibilidades de regulación para favorecer el abordaje de ellas, situación que obstaculiza el aprendizaje. No olvidemos que, luego de una instrucción escolar, emergen un conjunto de representaciones estudiantiles; entonces, ¿cuántas de ellas dan cuenta de la instrucción escolar deseada por el profesor para responder a los propósitos?

Según los antecedentes que hemos analizados, podemos sostener que existe fragmentación teórica sobre el conocimiento didáctico de la disciplina que se enseña, esto implica dificultad en el profesorado para transferir dicho conocimiento a situaciones de enseñanza auténticas que favorezcan la exploración de conocimiento y experiencias previas, diseño de estrategias en un marco de actividad científica escolar, identificación de obstáculos epistemológicos en la enseñanza, aprendizaje y evaluación de una noción científica, entre otros. Particularmente, la reflexión se sitúa desde el conocimiento contextual y no de los ámbitos del conocimiento didáctico del contenido disciplinar descritos por Valbuena (2007). Estas aseveraciones son, definitivamente, una real invitación a "mirar" qué y cómo abordamos las sesiones de práctica pedagógica con nuestro estudiantes, ¿hacia dónde apunta nuestra 'mira telescópica' cuando atendemos la formación de un profesor para el desarrollo de conocimiento profesional docente? 
Un segundo aspecto que nos parece preocupante es la sobrevaloración asignada por los profesores en formación al libro de texto como dispositivo de información e insumo de actividades de aprendizaje. Inmediatamente, cuestionamos la mermada capacidad "profesional" para la toma de decisiones vinculadas con la enseñanza. En ese contexto, Izquierdo (2007) sostiene que los profesores de Educación Básica conciben que un buen profesor sea quien domina aspectos curriculares y usa un libro de texto para orientar su enseñanza sobre un conocimiento. En ese plano, caracterizamos un profesor o profesora con un rol asumido más que deseado, situación que debemos discutir y, por cierto, problematizar; de lo contrario, estos noveles profesores adscriben a una cultura escolar que entiende que el libro de texto es importante en sí mismo: "Ahí está la materia que debo pasar; además, hay distintas actividades para desarrollar" (profesora en formación).

En relación a las actividades y los momento de una clase de ciencias, concluimos que se han invisibilizados los atributos que tipifican las actividades de aprendizaje; restringiendo posibilidades en el diseño y selección, pertinente, de tipos de actividades para promover el aprendizaje; además, queda de manifiesto una noción de aprendizaje más bien por apropiación y, en muchos casos, por sustitución de ideas más que por evolución gradual de ellas; como lo propone un diseño con secuenciación de actividades (Jorba y Sanmartí 1996).

Finalmente, la importancia de las preguntas en la clase de ciencias es relativa y no son consideradas en la reflexión sobre la planificación de clases. Este último es una invitación a redefinir tópicos y problemáticas que deben estar presentes en los talleres de reflexión docente inicial, de manera de cautelar que la discusión esté en estrecha relación con la sala de clases, valorando que la reflexión en la acción es un espacio fidedigno para promover conocimiento profesional y, específicamente, conocimiento didáctico.

\section{REFERENCIAS BIBLIOGRÁFICAS}

Bertelle, A.; Iturralde, C. y Rocha, A. (2006). Análisis de la práctica de un docente de Ciencias Naturales. Revista Iberoamericana de Educación, 37, 4, 1-9.

Couso, D. y Pintó, R. (2009). Análisis del contenido del discurso cooperativo de los profesores de ciencias en contextos de innovación didáctica. Enseñanza de las Ciencias, 27, 1, 5-18.

Fernández, M.T.; Tuset, A.M; De la Paz, G.; Leyva, A.C; Alvídrez, A. (2010). Prácticas educativas constructivistas en clases de ciencias. Propuesta de un instrumento de análisis, Revista Iberoamericana sobre Calidad, Eficacia y Cambio en Educación, 8, 1, 26-44.

Fuentealba, R. y Galaz, A. (2008). La reflexión como recurso para la mejora de las prácticas docentes en servicio: el caso de las redes pedagógicas locales en Chile. En Fuentealba, R y Cornejo, J. (Ed.), Prácticas reflexivas para la formación profesional docente: ¿Qué las hace eficaces? (pp. 141-167). Santiago: Ediciones Universidad Católica Silva Henríquez.

García, P. y Angulo, F. (2003). Un modelo didáctico para la formación inicial del profesorado de ciencias. Revista Interuniversitaria de Formación de Profesorado, 17, 001, 37-49.

Gorodokin, I. (2005). La formación docente y su relación con la epistemología. Revista Iberoamericana de Educación, 37, 5, 1-9.

García, A. (2009). La formación de profesores de ciencia a través de su interacción en Comunidades de Desarrollo Profesional. Tecné, Episteme y Didaxis, $N^{\circ}$ extraordinario $4^{\circ}$ Congreso Internacional sobre Formación de Profesores de Ciencia (pp. 77- 83).

Gutiérrez, A.; Valbuena, E. y Neira, L. (2009). La práctica reflexiva como estrategia para favorecer la construcción del conocimiento profesional del profesor de biología. Tecné, Episteme 
y Didaxis, $\mathrm{N}^{\circ}$ extraordinario $4^{\circ}$ Congreso Internacional sobre Formación de Profesores de Ciencia (pp. 942- 946).

Jorba, J. y Sanmartí, N. (1996). Enseñar, aprender y evaluar: un proceso de regulación continua. Propuestas didácticas para las áreas de Ciencias de la Naturaleza y Matemáticas. Madrid: MEC.

Labarrere, A. y Quintanilla, M. (2002). Análisis de los planos del desarrollo de estudiantes de ciencia. Efecto en el aprendizaje. Pensamiento Educativo, vol.30, 121-138.

Marcelo, C. (2002). Aprender a enseñar para la sociedad del conocimiento. Education Policy Análisis Archives, 10, 35.

Márquez, C. (2002). La comunicació multimodal en l’ensenyament del cicle del l’aigua. Tesis doctoral, UAB. Bellatera, Barcelona.

Márquez C.; Roca, M.; Gómez, A.; Sardá, A. y Pujol, R.M. (2004). La construcción de modelos explicativos complejos mediante preguntas mediadoras. Investigación en la Escuela, 53, pp.71-81

Moreno, J.M. (2006). Profesorado de Secundaria y calidad de la educación: un marco de opciones políticas para la formación y el desarrollo profesional docente. Profesorado. Revista de currículum y formación del profesorado, 10, 1. http://www.ugr.es/local/recfpro/Rev101ART3.pdf.

Orellana, M. (2008). La construcción de cuentos como proceso de modelización del entorno en la formación inicial de maestros de educación infantil desde la perspectiva de la semiótica social. Tesis de Doctorado para la Obtención del título de Doctora en Ciencias de la Educación, Pontificia Universidad Católica de Chile, Santiago: Chile.

Perafán, G (2005). Epistemología del profesor de ciencias sobre su propio conocimiento profesional. Enseñanza de las ciencias. Número extra, VII Congreso Internacional sobre Didáctica de las Ciencias, Barcelona.

Putnam, R. y Borko, H. (2000). El aprendizaje del profesor. Implicaciones de las nuevas perspectivas de la cognición. En B. Biddle, T. Good E I. Goodson (Eds.). La enseñanza y los profesores (I) La profesión de enseñar. Barcelona. Paidós.

Quintanilla, M. (2003). Hablar y construir la "Didáctica" hoy: Del modelo ingenuo transmisor, al modelo crítico, productor de conocimiento. Revista de Estudios y Experiencias, n.4, 69-82.

Ravanal, E. y Quintanilla, M. (2010). Caracterización de las racionalidades epistemológicas del profesorado de biología en ejercicio sobre la naturaleza de la ciencia. Revista Electrónica de Enseñanza de las Ciencias, 9, 1, 111-124.

Roca, M. (2005). Las preguntas en el proceso de enseñanza-aprendizaje de las ciencias. Educar, 73 - 80. Rodríguez, G.; Gil Flores, J. y García, E. (1999). Metodología de la investigación cualitativa. Granada: Aljibe.

Tardif, M. (2004). Los saberes del docente y su desarrollo profesional. Madrid: Narcea.

Vélaz de Medrano, C. (2009). Competencias del profesorado-Mentor para el acompañamiento al profesorado principiante. Revista de currículum y formación del profesorado, 13, 1, 209-229. 